\title{
The Demagnetization Field of Multidomain Grains
}

\author{
Ronald T. MERRILL* \\ Geophysics Program and Oceanography Department, \\ University of Washington, Seattle, U.S.A.
}

(Received June 20, 1977)

\begin{abstract}
The demagnetization 'constant', $N$, enters into all theoretical considerations of multidomain remanence. Although most workers believe $N$ to be related simply to the shape of the grain, no theoretical study of $N$ has previously been completed. It is shown in this paper that $N$ monotonically increases with increase in remanence in multidomain grains. Although $N$ can be approximated by a constant in the limit of small displacements of domain walls from their demagnetized sites, this constant critically depends on the domain geometry.
\end{abstract}

\section{Introduction}

The successes of paleomagnetism are based on the remarkable observation that many rocks retain a record of the direction of magnetization acquired hundreds to billions of years previously. The important works of NéEL on single domain theory (1949) and on multidomain theory (1955) remain at the foundation of most theoretical work which attempts to explain the remarkable stability of thermal remanent magnetization (TRM). The successes of Néel's theories are impressive. However, in recent years it has become increasingly clear that Néel's theories require modifications (for example Dunlop and West, 1969; Stacey and Banerjee, 1974; Dunlop and Waddington, 1975; Schmidt, 1976). One particular problem that has emerged can be illustrated by considering the mineral magnetite. It appears that the stable single domain size range for equant magnetite grains is very limited (BUTLER and BANERJEe, 1975). Yet larger grains appear to possess some properties that are more expected of single domain grains than multidomain grains (VERHOOGEN, 1959). Verhoogen suggests that the solution to this problem is that magnetization in screw dislocations in multidomain material acts like single domain grains. Although the details of VerHOOGEN's model (1959) appear unsatisfactory (SHIVE, 1969), the basic idea that 'something' inside large grains behaves in a single domain manner remains as a viable construct; this behavior is often referred to as pseudo-single domain behavior (STACEY, 1963; STACeY and BANERJEe, 1974). An alternative suggestion has been advanced by SCHмIDT (1973), who argues that two-domain grains behave very much like Néel's single domain grains, and very differently from much larger multidomain grains.

Therefore, it seems timely to reconsider the various assumptions of multidomain

* Contribution Number 973-Department of Oceanography. 
theory. This is done in the next section. Subsequently, one of these assumptions, that dealing with the demagnetization energy, is considered in detail.

\section{The Assumptions of Multidomain Theory}

At the onset we distinguish between 'true' multidomain theories and pseudo single domain theories. 'True' multidomain theories have been reviewed by DunLoP and WADDINGTON (1975); the critical distinction being that 'true' multidomain grains acquire their remanence through domain wall displacements. Pseudo single domain theories deal with nonuniformly magnetized grains which alledgedly acquire their stable remanence by mechanisms other than domain wall displacement. (For example, STACEY and BANERJEe, 1974, suggest that the remanence resides inside domain walls.) Hereafter, the modifier 'true' will be dropped, since its use might connote unintended implications of validity.

The usual assumptions made to obtain the magnetization, $J$, in multidomain grains are as follows: (1) The remanence is acquired and lost through domain wall motion: (2) Domain walls can be treated as being infinitesimally thick; (3) Equilibrium occurs in the presence of an external field, $h$, when the magnetic energy, $\frac{1}{2} \bar{J} \cdot \bar{h}$, is balanced by the demagnetization energy; (4) A remanence is acquired when domain walls are blocked by energy barriers, which are thought to be associated with crystal defects; (5) These energy barriers are associated with a microscopic coercivity, $H_{c}$, whose temperature dependence can be expressed as $\beta J_{S}^{n}$, where $\beta$ and $n$ are positive constants and $J_{S}$ is the saturation magnetization. An additional assumption is required when dealing with TRM: (6) The basic domain configuration is not strongly temperature dependent. That is, a grain with $p$ domains at elevated temperatures will still have $p$ domains at room temperature.

Many of these assumptions have been challenged. However, somewhat surprisingly, the nature of the demagnetization field has never been very critically examined. In all prior multidomain theories, the demagnetization field has been taken to be $-N J$; where $N$ is called the demagnetization factor, in analogy with single domain theory. This paper deals only with this assumption.

We briefly review multidomain theory to see how the demagnetization field assumption is used. This development is also useful later when we consider modified multidomain theories.

Dunlop and Waddington (1975) start with the following equations, which are consistent with the above assumptions. Equilibrium predicts that

or

$$
h-N J=-H_{c}=-\beta J_{S}^{n}
$$

$$
J=\frac{h+\beta J_{S}^{n}}{N} .
$$

Blocking of TRM can be defined in terms of $j$, which is defined by the equation:

$$
j \equiv \frac{J}{J_{S}} .
$$


Blocking occurs when there is no domain wall movement with decrease in temperature. That is,

$$
\frac{\mathrm{d} j}{\mathrm{~d} T}=0
$$

From Eqs. (1) and (4), we obtain:

$$
h=(n-1) H_{c} .
$$

Once blocking has occurred, the TRM increases only as the saturation magnetization increases. This can be taken into account by multiplying Eq. (2) by $J_{S o} / J_{S B}$, the ratio of saturation magnetization at room temperature to that at blocking temperature. Let $H_{c o} \equiv$ microscopic coercivity at room temperature $=\beta J_{S o}^{n}$ and $H_{c B} \equiv$ microscopic coercivity at blocking temperature $=\beta J_{S B}^{n}$. The Eq. (5) and the modified Eq. (2) can be used to obtain:

$$
J=\frac{n}{(n-1)^{(1-1 / n)}} \frac{h^{1-1 / n} H_{c o}^{1 / n}}{N}
$$

as given by Dunlop and WAdDIngton (1975).

There are two important results of Eq. (6). The first is that TRM is not linearly proportional to the external field. Although it is not clear what value of $n$ should be used, essentially all workers agree that $n$ is greater than 1 and less than 9 . The second result is that the intensity of TRM is inversely proportional to $N$.

\section{The Demagnetization Factor}

The first question that must be faced is: what is $N$ in Eq. (6)? Most workers believe $N$ to be related to the shape of the grain, in analogy with single domain theory. However, it is important to realize that $N$ is only well defined for single domain grains of certain simple shapes, such as ellipsoids of revolution. For non-uniformly magnetized grains, the demagnetization field will vary from location to location inside the grain. What is $N$ in such a case?

Insight into how one might define $N$ comes from the important work of KITTEL (1949), as reiterated by Chikazumi (1964, p. 209-211). Figure 1 shows the demag-
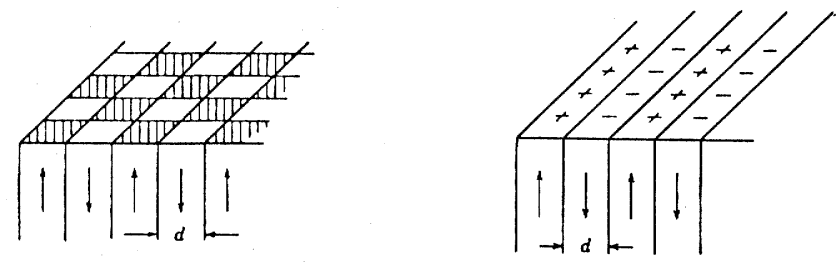

$$
E=2.37 \times 10^{4} J_{S}^{2} d
$$

$$
E=5.40 \times 10^{4} J_{S}^{2} d
$$

Fig. 1. The internal magnetic energy associated with bound surface charges for the checkerboard (left) and sheeted (right) domain structures. The examples given are for completely demagnetized states. 
netization energy associated with checkerboard and sheeted domain structure. Those results suggest that the so called 'demagnetization factor' might depend critically on the domain structure and not on the grain's shape. Note, however, that Kittel and Chikazumi have only treated domain structures in which there is no net magnetization. Therefore, no term associated with the particular demagnetization energies given in Fig. 1 appears in Eq. (1). We break the demagnetization energy up into two parts, $E_{o}$ and $E_{c}$. $E_{o}$ is the demagnetization energy associated with a completely demagnetized multidomain grain (examples are given in Fig. 1). $E_{c}$ is the extra demagnetization energy that results when a grain carries a remanence, that is, the extra energy that results from domain wall displacements from the zero field equilibrium position. It is the demagnetization field associated with this energy that should appear in Eq. (1). We define $N$ by the equation:

$$
E_{c}=\frac{1}{2} N J^{2}
$$

or

$$
N=\frac{2 E_{c}}{J^{2}}
$$

It is important to realize that, if we use the $N$ of Eq. (7) in Eq. (1), then we are still making a rather critical assumption. We are assuming that it is the average demagnetization energy for the entire grain that is important in TRM. This might not be the case if certain regions of a multidomain grain are blocked before others. In the latter situation, localized demagnetization energies must be considered. However, in the attempt to obtain a first order approximation for $N$, we neglect this last possibility.

We now set out to show that $N$ depends critically on the domain structure of a multidomain grain and that it increases with increase in mean wall displacement from the zero-field equilibrium position. We do this by considering the sheeted domain structure shown in Fig. 2. Several other configurations can be easily calculated and such calculations lead to changes in detail but not in principle. The important work of Kittel (1949) and ChiKaZumi (1964, p. 209-211) provide the necessary insight into the type of calculation required to determine $N$.
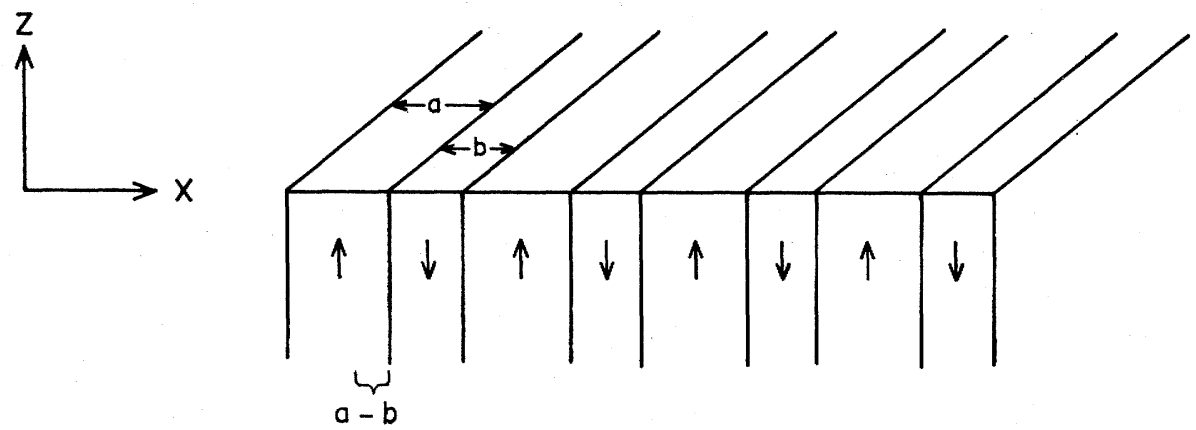

Fig. 2. Sheeted domain structure which exhibits a net remanence. This remanence is a result of displacement of every other domain wall by a distance $a-b$ from the completely demagnetized state. 
External to the grain, $\bar{V} \times \bar{h}=0=\bar{V} \cdot \bar{h}$. This implies that there exists a potential $\phi$, such that Laplace's equation holds:

$$
\nabla^{2} \phi=0 \text {. }
$$

Now, using MKS units, the magnetic induction, $\bar{B}$, is given by $\bar{B}=\bar{\mu}_{0}(\bar{h}+\bar{J})$, where $\bar{\mu}_{0}$ is the magnetic permeability in air. Using the fact that $\bar{V} \cdot \bar{B}=0$ everywhere, and that the magnetic bound charge is given by $\rho=\bar{\nabla} \cdot \bar{J}_{S}$, one has the boundary conditions:

$$
\begin{aligned}
& \left(\frac{\partial \phi}{\partial z}\right)_{z \rightarrow+0}=\frac{-\rho}{2 \mu_{0}} \quad 0<x<a, \\
& \left(\frac{\partial \phi}{\partial z}\right)_{z \rightarrow+0}=\frac{\rho}{2 \mu_{0}} \quad a<x<a+b .
\end{aligned}
$$

The following additional boundary conditions are also easily seen to be valid for the case under consideration:

$$
\begin{gathered}
\lim _{z \rightarrow \infty} \phi(z)=0 \\
\phi[x+(a+b) L]=\phi(x) \quad \text { for } L=\text { integer. }
\end{gathered}
$$

Eqs. (8) through (12) constitute a boundary value problem that is easily solved by separation of variables. The solution is:

where

$$
\phi=A_{0}+\sum_{m=1}^{\infty} A_{m} \cos \left(\frac{m \pi x}{a+b}\right) \mathrm{e}^{-\frac{m \pi z}{a+b}}+\sum_{m=1}^{\infty} B_{m} \sin \left(\frac{m \pi x}{a+b}\right) \mathrm{e}^{-\frac{m \pi z}{a+b}}
$$

$$
\begin{aligned}
A_{m} & =\frac{\rho(a+b)}{(m \pi)^{2} \mu_{0}} \sin \left(\frac{m \pi a}{a+b}\right) \\
B_{m} & =\frac{\rho(a+b)}{(m \pi)^{2} \mu_{0}} \cos \left(\frac{m \pi a}{a+b}\right) .
\end{aligned}
$$

$A_{0}$ is a constant of little consequence in this problem and may be dropped ( $\phi$ uniquely defines the magnetic problem, external to the grain, within an arbitrary constant).

The next step in the calculation utilizes the important observation of KitTeL (1949) that one can obtain the self-demagnetization energy by calculating the surface energy. The surface energy, per unit area is:

$$
\dot{E}=\frac{1}{2} \rho \phi_{z=0}
$$

It is easy to show, by using $\nabla \cdot \bar{J}_{S}=\rho$ and a 'pill-box' approach at the boundary $z=0$, that the surface charge per unit area is equal to $J_{S}$ for the domain configuration of Fig. 2. Therefore, the average self-demagnetization energy per unit area is:

$$
\begin{aligned}
E= & \sum_{m=1}^{\infty} \frac{J_{S}^{2}}{2 \mu_{0}} \frac{(a+b)}{(m \pi)^{2}} \sin \left(\frac{m \pi a}{a+b}\right)\left[\frac{1}{a} \int_{0}^{a} \cos \left(\frac{m \pi x}{a+b}\right) \mathrm{d} x-\frac{1}{b} \int_{a}^{a+b} \cos \left(\frac{m \pi x}{a+b}\right) \mathrm{d} x\right] \\
& +\sum_{m=1}^{\infty} \frac{J_{S}^{2}}{2 \mu_{0}} \frac{(a+b)}{(m \pi)^{2}} \cos \left(\frac{m \pi a}{a+b}\right)\left[\frac{1}{a} \int_{0}^{a} \sin \left(\frac{m \pi x}{a+b}\right) \mathrm{d} x-\frac{1}{b} \int_{a}^{a+b} \sin \left(\frac{m \pi x}{a+b}\right) \mathrm{d} x\right]
\end{aligned}
$$


or

$$
\begin{aligned}
E= & \sum_{m=1}^{\infty}\left\{\frac{J_{S}^{2}}{2 a b} \frac{(a+b)^{3}}{\mu_{0}(m \pi)^{3}} \sin ^{2}\left(\frac{m \pi a}{a+b}\right)-\frac{J_{S}^{2}(a+b)^{2}}{2 a b \mu_{0}(m \pi)^{3}}\right. \\
& \left.\times\left[(a+b) \cos ^{2}\left(\frac{m \pi a}{a+b}\right)+\frac{\left(b-a[-1]^{m}\right)}{a b} \cos \left(\frac{m \pi a}{a+b}\right)\right]\right\} .
\end{aligned}
$$

It is clear from Eq. (18) that the average internal self-demagnetization energy is dependent on the domain configuration. Following the examples provided by the earlier theories for multidomain grains we would like to express the self-demagnetization energy in terms of the remanent magnetization, $J$, which is linearly proportional to $J_{S}(a-b)$ (see Fig. 2). If any of the previous multidomain theories were correct, then the energy should be given by $\frac{1}{2} N J^{2}$, where $N$ depends solely on grain shape. As seen from (18), this is not the case. To see this more clearly, and to obtain simpler relationships to work with, we consider two extreme limits for Eq. (18).

We consider first the case where there is only a small displacement of the domain walls from the zero-field equilibrium position. This corresponds to the case of small remanence in multidomain grains. In this case, $a /(a+b) \simeq \frac{1}{2}$, etc. and (18) reduces to

$$
E \simeq \frac{J_{S}^{2}(a+b)^{3}}{2 \mu_{0} \pi^{3} a b} \sum_{m \text { odd }} \frac{1}{m^{3}}
$$

We further reduce Eq. (19), by noting that $a$ and $b$ are not independent of each other. We write:

$$
\begin{aligned}
& a \equiv d+\varepsilon \\
& b \equiv d-\varepsilon
\end{aligned}
$$

where $\varepsilon$ is the distance the domain wall is displaced and $d$ is the zero-field equilibrium dimension of the domain (see Figs. 1 and 2). Therefore $(a+b)=2 d$. 1/ab can be approximated by the first few terms in a Taylor's expansion for small $\varepsilon$ :

$$
\frac{1}{a b}=\frac{1}{d^{2}-\varepsilon^{2}} \simeq \frac{1}{d^{2}}+\frac{2 \varepsilon^{2}}{d^{4}}+\frac{12 \varepsilon^{4}}{d^{6}} .
$$

We note that the displacement $\varepsilon$ is linearly proportional to the remanence (The remanence increases as $2 \varepsilon$, since, when the magnetization increases in $a$, it decreases in $b$ ). We write:

$$
\varepsilon=c \frac{J}{J_{S}}
$$

where $c$ is a constant. Substituting these results into Eq. (19) and also using the result (CHIKazUmI, 1964, p. 211) that $\sum_{m \text { odd }} \frac{1}{m^{3}} \simeq 1.0517$, we get

$$
E \simeq \frac{4.2 J_{S}^{2} d^{3}}{\mu_{0} \pi^{3}}\left[\frac{1}{d^{2}}+\frac{2 \varepsilon^{2}}{d^{4}}+\frac{12 \varepsilon^{4}}{d^{6}}\right]
$$

or

$$
E \equiv E_{0}+\frac{\left(A+B \varepsilon^{2}\right) J^{2}}{2}=E_{0}+E_{c}
$$


where $E_{0}, A$ and $B$ are constants that depend on the domain structure, as given above. In the limit that $\varepsilon=0$ (this implies $J \rightarrow 0$ ), $E=E_{0}=4.2 J_{S}^{2} d / \mu_{0} \pi^{3}$, a result obtained by Kittel (1949) and ChiKazumi (1964, p. 211). (The above number is twice that given by these authors, simply because we have obtained the sum of the average energy in both domains.) This consistency provides some confidence that no mathematical errors have occurred in the derivation of Eq. (18). From Eqs. (7) and (22) we obtain the demagnetization factor for small wall displacements.

$$
N=\left(A+B \varepsilon^{2}\right) \text {. }
$$

\section{Discussion and Conclusions}

Equation (23) can be used to illustrate some important conclusions. First, in the limit of very small domain wall displacements, the demagnetization factor can be taken as a constant, independent of wall displacement. Therefore, the theory leading to Eq. (6) is correct only for small wall displacements. However, small wall displacements are probably to be expected for weak-field TRM. Equally important, the factor $N$ is related to domain structure and not to the shape of the grain. The grain's shape will enter only to the extent that it affects the domain structure.

$N$ increases with further wall displacement. One can calculate what $N$ is close to saturation. (Note, however, that care must be used, if the preceding equations are used to do this. For example, the second and fourth terms in Eq. (17) have factors going to infinity and to zero, as $b$ approaches zero. Care must be used so that the Moore-Smith convergence criterion is not violated.) Complicated mathematical arguments are unnecessary for the limit when saturation is approached, since the grain approaches a single domain configuration. However, contrary to what might be expected at first glance, $N$ is still a function of $\varepsilon$, close to the limit. The limiting value of $\varepsilon$ is related to the shape of the domain ' $a$ '. Effectively, what is happening is that the effect of the magnetization in the $b$ domain becomes infinitesimally small as one approaches the limit. Therefore, the change in $N$ close to the limit is well approximated by considering the change in shape of the $a$ domain. In this limit $N$ is the 'shape' demagnetization factor associated with a single domain grain with shape identical to that of domain $a$.

The above results suggest that the following theorem may be valid: $N$ is a monotonically increasing function of $\varepsilon$. Proof of this theorem is difficult, since it is invalid to extrapolate from particular models of domain geometry to the general situation. Nevertheless, the theorem appears reasonable, even if it remains a difficult one to prove rigorously.

Because $N$ appears to be an increasing function of $\varepsilon$, the acquisition of remanence will not vary simply as a function of the external field raised to some power $\left(h^{1-1 / n}\right.$, as given in Eq. (6)). This prediction is consistent with the $\log -\log$ plots of $J$ versus $h$ as found by Dunlop and Waddington (1975) and by DAY (this issue). However, this result does not explain the apparent remarkable consistency between results for alleged single domain TRM and multidomain TRM (DAY, this issue). 
In conclusion, the demagnetization factor has been found to depend on domain structure in grains with small wall displacements and on grain shape in grains close to saturation. Previous multidomain theories correctly treat the demagnetization field only in the limit of very small wall displacements. It is difficult to predict the precise way the demagnetization factor will increase in real situations where closure domains, etc., are present. Nevertheless, it seems reasonable to expect that $N$ will generally be a monotonically increasing function of the mean distance of domain wall displacement from the zero field equilibrium configuration.

Critical reviews by Drs. Robert Butler, David Dunlop, Mike Fuller and Frank Stacey greatly improved the quality of this paper. Support for this work came from NSF grants OCE-01541 and OCE 75-21002.

\section{REFERENCES}

BUtLER, R.F. and S.K. BANERJEE, Theoretical single-domain grain size range in magnetite and titanomagnetite, J. Geophys. Res., 80, 4049-4058, 1975.

Chik azumi, S., Physics of Magnetism, pp. 554, John Wiley and Sons, New York, 1964.

DAY, R., TRM and its variation with grain size, J. Geomag. Geoelectr., 29, 233-265, 1977.

Dunlop, D.J. and E.D. WADDington, The field dependence of thermoremanent magnetization in igneous rocks, Earth Planet. Sci. Lett., 25, 11-25, 1975.

DunLOP, D.J. and G.F. WeSt, An experimental evaluation of single domain theory, Rev. Geophys., 7, $709-758,1969$.

Kittel, C., Physical theory of ferromagnetic domains, Rev. Mod. Phys., 21, 541-583, 1949.

NÉEL, L., Théorie du traînage magnétique des ferromagnêtiques en grains fins avec applications aux terres cuites, Ann. Géophys., 5, 99-136, 1949.

NÉEL, L., Some theoretical aspects of rock magnetism, Adv. Phys., 4, 191-243, 1955.

SCHMidT, V.A., A multidomain model of thermoremanence, Earth Planet. Sci. Lett., 20, 440-446, 1973.

SchmidT, V., The variation of the blocking temperature in models of thermal remanences (TRM), Earth Planet. Sci. Lett., 29, 146-154, 1976.

SHIVE, P., The effect of internal stress on the thermoremanence of nickel, J. Geophys. Res., 74, 3771$3780,1969$.

STACEY, F.D., The physical theory of rock magnetism, Adv. Phys., 12, 45-133, 1963.

Stacey, F.D. and S.K. BAnerJee, The Physical Principles of Rock Magnetism, pp. 195, Elsevier, Amsterdam, 1974.

VeRHOOGEN, J., The origin of thermoremanent magnetization, J. Geophys. Res., 64, 2241-2449, 1959. 